\title{
Primary Nerve Repair with or without Tibialis Posterior Transfer for Management of Common Peroneal Nerve Palsy
}

\author{
Mohamed Abd-El Aziz Mohamed Ali MD ${ }^{1 *}$, Mohammed Mansour MD $^{2}$, Nagy Fouda MD $^{3}$ \\ ${ }^{1,2}$ Assisstant Professors of Orthopedic Surgery, Zagazig University, Egypt \\ ${ }^{3}$ Lecturer of Orthopedic Surgery, Zagazig University, Egypt
}

*Corresponding Author: Mohamed Abd-El Aziz Mohamed Ali MD, Assisstant Professor of Orthopedic Surgery, Zagazig University, Egypt.

\begin{abstract}
Objective: As a result of high risk of failure rate with cases of common peroneal nerve (CPN) palsy with nerve gap defect more than 6cms when managed by sural nerve graft alone so we preferred to proceed directly to tibialis posterior (T P) transfer and sural grafting in one step and evaluate results of common peroneal nerve lesions (CPN) displaying nonfunctional regenerative signs. When explored and Intraoperative decision making to manage the lesions either by primary microsurgical nerve repair or one step primary nerve repair and tibialis posterior transfer reconstructive surgery to provide the patient the best chances of sensory recovery and useful motor functional outcomes .
\end{abstract}

Methods: Forty two patients with common peroneal nerve lesions were managed in Zagazig university hospital (2013 - 2017), every cases of CPN palsy before surgery, we mentioned the potential of TP transfer during the surgery in one step. 16 cases primary microsurgical nerve repair was done by external neurolysis or internal interfasicular neurolysis. 15 Cases explored, neuroma excision and nerve graft if gap nerve defect less than $6 \mathrm{~m}$ with good number of motor end plate evidenced by MUNE of anterior and lateral leg muscles. (11) Cases managed by autologous sural nerve graft and tibialis posterior transfer if gap nerve defect more than $6 \mathrm{~m}$ with few number of motor end plate evidenced by MUNE of anterior and lateral leg muscles and viable sensory receptor evidenced by SEVP.

Results: The follow up range (20 - 60) months, patients were satisfied with motor recovery grade (4) and (5) of BMRC, so in (16) cases neurolysis their results were excellent and satisfied in (15/16) (92.5\%). (15) Cases gap defect less than $6 \mathrm{cms}$ were managed with primary nerve repair with sural nerve graft length (3-9 cms) their results were good and satisfied in (12/15) (80\%) (11) cases of nerve gap defect more than $6 \mathrm{~cm}$ were managed by one step T P tendon transfer and primary nerve repair with sural nerve graft length $(9.5-12 \mathrm{cms})$ their results were excellent and satisfied in (10/11) (90.9). In our study (42) cases with CPN lesions were managed by primary nerve repair or primary nerve repair and secondary TP tendon transfer in one step their motor results were excellent and satisfied in (37/42,88.1\%), regarding sensory recovery of CPN palsy after our procedures (20) cases described complete sensory recovery (47.6\%) and considered excellent results, (17) cases improved sensory deficit (40.4\%) and regarded as good results, (5) cases with unchanged sensory deficit $(11.9 \%)$ and considered bad result, good and excellent results were considered by the patients as satisfied results. So in our study (37) cases reported satisfied sensory recovery after our procedures (37/42 88.1\%). Neuropathic pain was recorded in (16) cases of CPN palsy, after our surgical procedures (6) cases showed complete regression, (7) cases improved with reduction of neuropathic pain, (3) cases recorded as unchanged neuropathic pain and referred to pain control clinic to manage them (13/16, 81.2\%).

Significant correlation between age, causative mechanism of injury, time of injury and surgical procedure and motor and sensory recovery $(P=0.002)$.

Conclusion: In our study we provided excellent improvement in motor and sensory function recovery of CPN palsy and no patient had deteriorated. The overall results of all procedures to manage (42) patients of CPN lesions were satisfied and excellent in (37/42, 88.1\%). Sensory recovery of CPN palsy were good and excellent results and considered by the patients as satisfied results (37/42, 88.1\%). Neuropathic pain after our surgical procedures showed improved with reduction of neuropathic pain, (13/16, 81.2 \%). Factors affect quality of recovery after reinnervation were accuracy of fascicular matching, length of gap defect after neuroma resection, number of channels in grafts, the number of axons reaching the correct target end organs and its development and myelination and attention to the time interval between starting injury and surgery and graft length used and tendon transfer all effect on functional outcome.

Keywords: common, Peroneal, Tibialis, transfer, nerve repair 


\section{INTRODUCTION}

Across all series, the common peroneal nerve has fearless potential for functionally reinnervation after primary nerve repair when compared with other nerves like radial or tibial nerve and was resulted from its mixed sensorimotor nature, small amount of fascicles, fewer and larger bundles and less connective tissue and less adipose tissue in epineurium than tibial division and lager nutrient vessels on nerve surface so more exposure to injury and compression. Its superficial site at the head of fibula increases its liability to traumatic injury [1].

The motor functional unit formed of (single motor neuron and all muscle fibers innervated by it that follow all or non-law, innervation of muscles an axons of cell body in anterior of spinal cord branches near its ends to enervate several muscle fiber, forming neuro muscular junction called motor end plate) and sensory unit (peripheral sensory receptors in the skin and deep receptors in muscles), in injury of CPN must be not concentrate on site of lesion only but overview, as effects of injury produce pathology in muscle fibers, intra fusal muscle spindles, Golgi tendon organ and sensory receptors. Denervation of anterior and lateral leg compartments leads to muscle atrophy so early surgical repair, electrical stimulation and early motion prevent muscle atrophy by maintaining metabolic capacity of muscle fibers [2].

In CPN injury, the effect not localized on site of lesion but there are changes in proximal and distal stumps of the nerve which increase by time if regeneration of axons into distal stump fails or long delayed changes in the end organs and proximal neurons which over time become irreversible, in denervated muscles motor end plates atrophy and disappear with time started at 3 months and after 3 years irreversible fibrotic changes, cutaneous sensory end organs atrophy more slowly to occur after 5 months. In avulsion CPN from muscles direct muscular neurotization by implantation of fiber into paralyzed muscle hope for development of end plates in response to the entry motor axons [3].

Nerve fiber not only transmit impulses but also serve as anatomical connection between the nerve cell body and its end organs, maintained by axonal transport system, substances synthesized in cell body "proteins" are transported from cell body to the periphery and take place at speeds that vary from $(1-40 \mathrm{~mm}$ per day) which is important in nerve regeneration at repair site [4]. The most frequently affected between lower limb nerve lesions. Functional outcome after reconstruction is usually disappointing when compared with other injured nerves (radial nerve). Julia et al stated that their experience with common peroneal nerve lesions and management strategy with Functional useful result $(M \geq 4)$ was achieved in cases where reconstructed by neurolysis external or internal in nerve compression or adhesions but outcome results is decreased in cases after end to end suture or nerve grafted $<6 \mathrm{~cm}$ and nerve surgery results are poor in nerve grafted $>6 \mathrm{~cm}$.

Relative strength percentage of dorisiflexors of ankle tibialis anterior (5.6), extensor digitorum longus (1.7), extensor halluces longus (1.2) peroneous tertious (0.9) and invertors strength percentage tibialis posterior (6.4). Ankle motion occurs in sagittal plane flexion $\left(40-55^{\circ}\right)$ and its maximum is toe off and extension $\left(10-20^{\circ}\right)$ and its maximum in stance phase. So tibialis posterior transfer is suitable one [5]. Electro diagnosis studies (electromyogram 'EMG'), nerve conduction studies (sensory nerve action potential 'SNAP' and motor nerve action potential 'MNAP. EMG were performed as base line and useful in follow up postoperative recovery, examined muscle electrical activity units and evidence of degeneration [fasciculation or fibrillation] or regeneration [muscle action potential]. Nerve conduction and (EMG) were useful in making diagnosis, any drop in amplitude more than $20 \%$ is significant [6].

Motor unit number estimation (MUNE), electromyography (EMG) and compound muscle action potential (CMAP) amplitude it was evident that early reduction (MUNE) was before detectable decline of motor activity, the early reduction (MUNE) is significant as it suggests that molecular changes associated with injury and leading to delayed neurodegeneration so indicate early intervention to prevent poor results with the nerve repair. (MUNE) offer the advantage of early quantitative detection of motor unit abnormalities when both the clinical and electro diagnostic parameters are normal and provides unique tool to estimate the number of axons innervations a muscle. (MUNE) is computed from the ratio between maximum CMAP divided by the average surface motor unit potential [7]. 
Role of superficial peronial sensory potential and high resolution ultrasonography in confirmation of common peronial mono neuropathy at fibular neck [8].

Patients and methods: in our study (42) cases were managed by intraoperative decision of primary nerve repair or primary and tibialis posterior transfer reconstructive surgery of common peroneal nerve lesions. Our study was based on preoperative clinical evaluation of all cases, sensory and motor deficits, causative mechanism of injury, age at surgery, time interval between lesions and time of surgery, radiological, electro physiological studies (motor evoked membrane potential (MEMP), sensory evoked membrane potential (SEMP), electromyography (EMG) and nerve conduction study (NC), motor grading by British Medical Research Council (BMRC) preoperative muscle power grading from M0 (no contraction palpable) to M5 (full range of motion with maximum force), the strongest extensor ankle is tibialis anterior which is most active during stance phase from heal strike to foot flate and allow foot clearance from the floor during swing phase so management of CPN palsy is critical. Superficial sensory examination, neuropathic pain, pain scoring visual analog scale for pain (VAS) $(0-10)$. All [42] cases were examined preoperatively and postoperatively by clinical muscle grading according to BMRC. EMG is a useful prognostic tool as it predicts muscle reinnervation before any other test, early appearance of reinnervation does not prove that full function reinnervation, absence of reinnervation at 3 months indicated exploration but presence innervation seemed to confused clinical outcome as motor activity in a muscle is not predicting of acceptable level of motor recovery. SNAP tested sensory receptors, as electrical normal or not. MNAP velocity tested the motor unit, as normal or not. Preoperative motor unit number estimation was useful to test the extent of motor axon regeneration. Preoperative sensory action potential of the ipsilateral sural nerve is mandatory to decide, if we will take ipsilateral or contralateral sural graft as in some cases sural nerve receive sensory input from lateral cutaneous sural nerve from the injured peroneal nerve. Lesion of most cases of CPN nerve was managed after 2 months except one case in the first day of injury so the ipsilateral lateral cutaneous nerve undergone Wallerian degeneration so the chronically axonotomiesed Schwann cells in the graft were less receptive to the regenerating axons so in this cases the ipsilateral graft is not good conduit.

\section{Surgical TeChNique}

All cases were anaesthetized under general anaesthia without muscle relaxant or short acting muscle relaxant to facilitate intraoperative direct nerve stimulator and EMG and were positioned in prone. Our target from exploration of the common peroneal nerve is to make diagnosis and strategy of reconstruction of it. Intraoperative microscope, direct nerve stimulator and EMG were available for all cases. The lower limb was free draped from hip to toes so allowed access to the entire common peroneal nerve from sciatic nerve at hip joint origin of the nerve to its muscular branches to anterior and lateral compartment of the leg to exclude apparent anatomy and double level lesion. CPN located in adipose connective tissue explore proximal as its origin from sciatic nerve and distal till the hillum of muscle supplied by deep PN and superfascial PN Now dissection of scar tissue start with clear planes were developed this avoid injury of the nerve by prisk direct dissection into soft tissue scar the course of the nerve is identified, intraoperative direct.

Nerve stimulator and EMG to evaluate the nerve conductivity after complete isolation from scare tissue and the patient recovered from muscle relaxant now recording the response of stimulation of each fascicle of the CPN clinically proximal to the lesion to prove absence or presence of regeneration nerve with evoked (NAP) indicate neurolysis. We used direct nerve stimulation at $0.5,1.0,2.0$ main lesion in continuity to decide to proceed with neuroma resection and grafting, neurolysis or tendon transfer. Cases with contraction muscle leads to move the joints if ankle dorsiflexion and toes extension indicate external neurolysis of deep PN and eversion of the foot so spontaneous recovery is suspected of superfascial PN, if the conduction across the lesion showed lesser decrease in the amplitude of muscle action potential of internal neurolysis is indicated until individual fascicles identified. Microsurgical external neurolysis was done by freeing the nerve from epineural fibrosis and constricting and compression agents. Microsurgical internal neurolysis were done by exposure of fascicles by epineurotomy and separate individual fascicles by excision of interfasicular scare tissue. After both types of 
neurolysis intraoperative nerve stimulation were done to be sure that the nerve was conducting with muscle contraction moving joints if not, excision and graft the site of lesion was preferred. If the drop in amplitude across the neuroma was more than $(50 \mathrm{ma})$ the neuroma was excised until healthy fascicles structure, unless adequate healthy CPN fiber present grafting is useless, distal dissection of the neuroma until healthy distal stump was identified, all dissection of neuroma and proximal and distal cut ends of CPN was done under high magnification microscope. The gap between ends was measured, no end to end repair was done as our target was tension free micro sutures to avoid repair site failure and disadvantage of fibrosis in healing. Nerve graft length must (10-15\%) longer than the gap defect, in our study we used graft length $(15 \%)$ longer to avoid latter contraction of the graft during healing, the number of neural channel provided by graft must be the same number of nerve fascicles to provide and restore continuity according to anatomical relationship sensory fascicle to sensory one and motor one to motor fascicle so enough sural nerve graft length and number must be available to cover the face of nerve ends and lie relaxed completely after fixation at each ends so for example gap defect $6 \mathrm{~cm}$ needs $9 \mathrm{~cm}$ sural graft length. We reconstructed selectively CPN to deep peroneal and to superfascial peroneal with exposure of the hilum of tibialis anterior and peroneus longus to exclude double level lesion and nerve avulsion from muscle which were found in (2 cases) to ensure good anatomical repair. Why sural nerve used as nerve graft as its considerable length, minimal branching so better candidate axonal conduction. If the gap defect more than $6 \mathrm{~cm}$, we did interposition sural nerve graft and tibialis posterior transfer in one step. In our study (6) cases ipsilateral and (20) cases grafted from contralateral sural nerve. In (2) cases of CPN palsy ware avulsion of deep peroneal nerve from tibialis anterior hilum muscle were managed by reimplantation of the nerve into the muscle to stimulate motor end plate to regenerate. In our study cases of CPN palsy with (nerve gap defect more than $6 \mathrm{~cm}$, nerve was avulsed from muscle hilum, old age patient, if delay between pathology and surgery and if lesion more than 10 months ago and present with complete motor loss without any regenerative sign, in our study were managed by simultaneous nerve graft and tibialis posterior transfer. In these cases after exploration of CPN,
First step was TP transfer, then second step was nerve graft. Prerequested for tibialis posterior transfer full toes and ankle passive range of motion, cases with moderate passive ankle dorsiflexion need for Achilles tendon manual rehabilitation therapy before surgery, foot intrinsic muscles, FDP, FHL, TP. Gastrocnemius muscles with normal function and strength. If equines deformity was present myotendenous fractional lengthening or tendoachilis lengthening and posterior ankle capsulotomy was necessary. The patient was turned supine short transverse incision on the navicular bone TP tendon was sectioned and stay suture, muscle belly was exposed through posteromedial incision, then anterolateral incision proximal to ankle then the tendon was drawn anteriorly from interosseous membrane lateral to subcutaneous surface of the tibia, then passed deep to the extensor retinaculum, we splinted TP tendon into two parts formed Y shaped, sutured to TA tendon and peroneous tertious with equal tension when peroneous tertious was absent or small size, we fixed this part of TP tendon to the bone so tenodesis was developed. The tension of transfer, the foot was close to neutral and in neutral balance between inversion and eversion. After closure of wound of transfer, the patient was turned prone to complete sural nerve graft under microscopic magnification. Surgical procedures (I) external neurolysis were (9) cases, (II) internal neurolysis (7) cases, (III) neuroma and nerve lesion excision with gap defect between nerve ends (less than $6 \mathrm{~cm}$ ) were (15) cases were managed by sural nerve graft length range (3-9 cms), (IV) neuroma and nerve lesion with gap defect between nerve ends (more than $6 \mathrm{~cm}$ ) were (11) cases were managed by one step tibialis posterior tendon transfer and sural nerve graft, nerve graft length range $(9-12 \mathrm{cms})$.

\subsection{Post-Operative Follow Up}

TP transfer as 2ry reconstruction after 1ry microsurgical repair of CPN or as one step with 1ry repair the transfer was protected in below knee cast for 6 weeks after that cast removed, stitches were removed, drop foot orthoses were used, we waited for 18 months during it we followed up cases 1ry repair with progression of tinel sign, muscles contraction and nerve conduction studies, all cases with muscle grade 3 recovery with orthoses, Active exercise were started but tendon transfer were protected for 6 month 
Primary Nerve Repair with or without Tibialis Posterior Transfer for Management of Common Peroneal Nerve Palsy

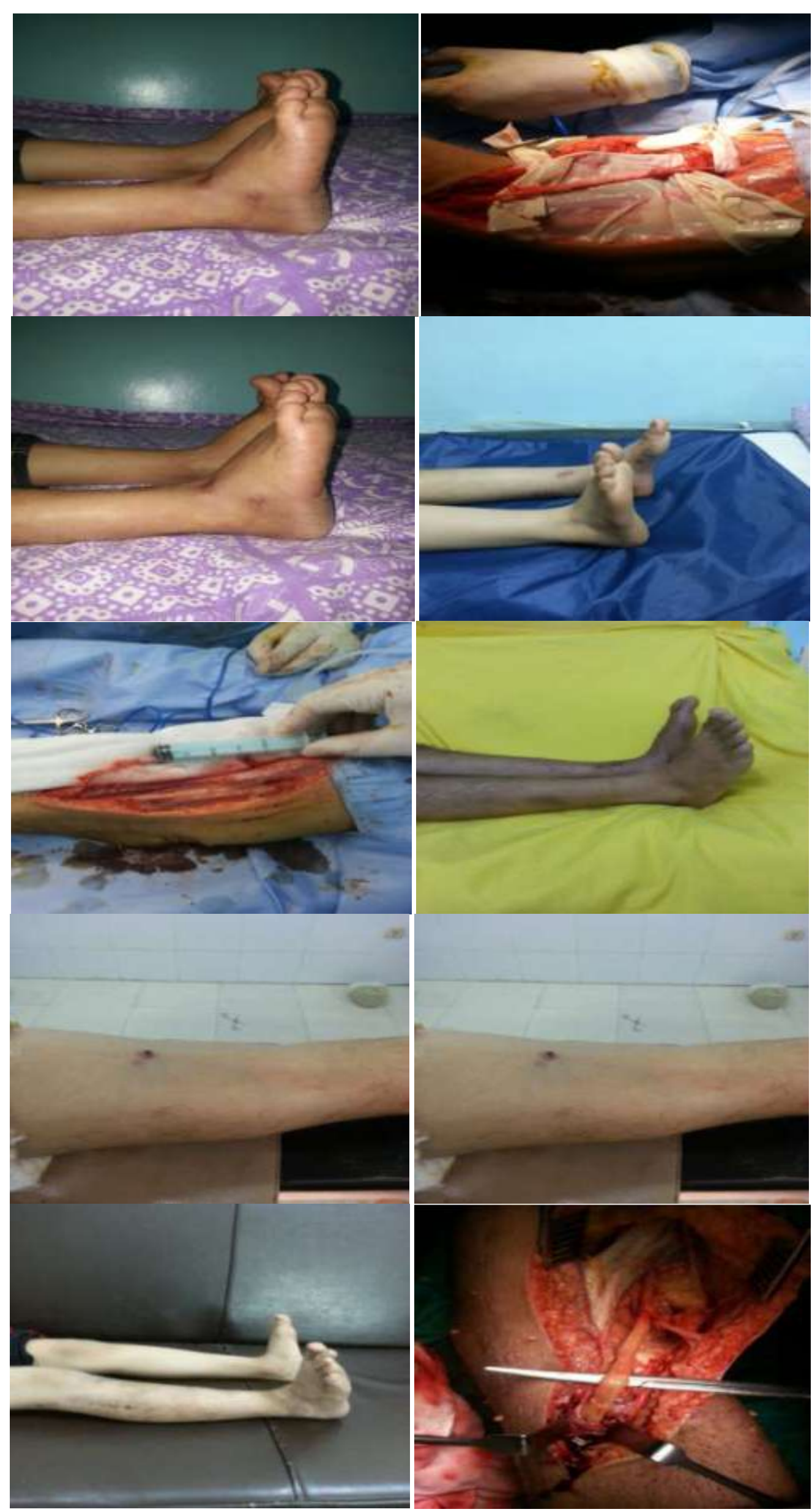




\section{Results}

Age range (5-52 years), sex (24) male and (18) female, side (22) left and (20) right, mechanism of CPN palsy were Osteochondroma (4), ganglion (2), compound fracture (9), stap wound (6), gun shoot(5), knee arthrolysis surgery (1), schwannoma (3), donkey bite (4), calcified hematoma (1), knee dislocation (5), high tibial osteotomy (2). Time from lesion to surgery range (one day -11 months). Sensory affection were (16) cases with neuropathic pain, (28) cases with hyposethia, (14) cases with anesthesia. Motor affection according to BMCR range from (0 - 3 grade). According to the preoperative clinical and electro diagnostic studies, intraoperative exploration finding and intraoperative nerve action potential (NAP) and surgical procedures cases were classified into three groups: Group (A) were (16) cases with CPN nerve lesion in continuity with regenerative potential were managed by external or internal neurolysis (16/42) with useful functional recovery of ankle dorsiflexion and foot evertion, BMRC range (4-5) with excellent results of $(14 / 16,87.5 \%)$. External neurolysis were (9) cases with (6) cases grade (4) of BMRC and (3) cases with grade (5) so useful functional outcome were $(9 / 9,100 \%)$. Internal neurolysis were (7) cases with one case recovery grade (3) was managed by secondary $\mathrm{T} \mathrm{P}$ transfer after 18 months follow up, (4) cases recovery grade (4) and two cases recovery grade (5) so useful functional outcomes were (6/7, 85.7\%). Group (B): (15) cases with CPN palsy with no signs of reinnervation potential were managed by exploration of CPN and sural grafting for gap defect (less than $6 \mathrm{cms}$ ), (3) cases recovery grade (3) these cases were managed by secondary TP transfer, (10) cases recovery grade (5), (2) cases recovery grade (4) so useful functional outcomes were (12/15, $80 \%$ ). Group (C): (11) cases with no signs of regenerative potential of CPN palsy were managed by exploration, sural grafting for nerve gap defect more than $6 \mathrm{cms}$ and simultaneous $\mathrm{T}$ $\mathrm{P}$ transfere in one step, one case recovery grade (3) this case was managed by arthroscopic ankle arthrodesis, (10) cases motor recovery grade (5) with useful functional outcomes $(10 / 11,90.9 \%)$. In our results, patients were satisfied with motor recovery grade (4) and (5) of BMRC, so in (16) cases of C P N lesions were managed by internal and external neurolysis their results were excellent and satisfied in $(15 / 16,92.5 \%)$, in (15) cases of CPN lesions with nerve gap defect less than $6 \mathrm{cms}$ were managed with primary nerve repair with sural nerve graft length $(3-9 \mathrm{cms})$ their results were good and satisfied in (12/15, $80 \%$ ), in (11) cases of CPN lesions with nerve gap defect more than $6 \mathrm{~cm}$ were managed by one step T P tendon transfer and primary nerve repair with sural nerve graft length $(9.5-12 \mathrm{cms})$ their results were excellent and satisfied in $(10 / 11,90.9)$, in our study (42) cases with CPN lesions were managed by primary nerve repair or primary nerve repair and secondary $\mathrm{T} P$ tendon transfer in one step were excellent and satisfied in $(37 / 42,88.1 \%)$. The follow up range (20 - 60) months regarding sensory recovery of CPN palsy after our procedures (20) cases described complete sensory recovery $(47.6 \%)$ and considered excellent results, (17) cases improved sensory deficit (40.4\%) and regarded as good results (5) cases with unchanged sensory deficit (11.9\%) and considered bad result. Good and excellent results were considered by the patients as satisfied results $(37 / 42,88.1)$. In group A (8) cases showed excellent sensory recovery result and (8) cases good recovery, all cases were satisfied, $100 \%$. In group B (9) cases reported excellent (4) cases good sensory recovery result $(13 / 15,86.7 \%)$ of cases were satisfied and (2) cases bad result and in group C (3) cases excellent sensory recovery, (5) cases good $(8 / 11,72.7 \%)$ of cases were satisfied and (2) cases bad sensory recovery. So in our study, (37) cases reported satisfied sensory recovery after our procedures (37/42, $88.1 \%$ ). Preoperative neuropathic pain was recorded in (16) cases of CPN palsy, after our surgical procedures (6) cases showed complete regression, (7) cases improved with reduction of neuropathic pain, (3) cases recorded as unchanged neuropathic pain and referred to pain control clinic to manage them $(13 / 16,81.2 \%)$.

\section{DisCUSSION}

Every case of CPN palsy before surgery, we mentioned the potential of TP transfer during the surgery in one step, if the age of the patient old, in sever mechanism of palsy, in long time per months between palsy and surgery, if clinical and electrophysiological study findings reported complete motor loss without regenerative signs and preoperative motor unit number estimation a useful test to test the extent of motor axons regeneration was low. Kim et al (2004) reported 316 cases, their results were good functional outcome in (88\%) after neurolysis, after end to end suture (84\%) and grafted peroneal nerves were functional 
outcomes (41\%) and they demonstrated the longer grafts length provided a worse outcome, patient with grafts less than $6 \mathrm{cms}(75 \%)$, cases with grafts from (6-12) results (38\%) and cases with grafts length longer than $(12 \mathrm{cms})$ results $(16 \%)$ obtained in useful function dorsiflexion of the foot. Kim et al used muscle grading system with useful dorsiflexion is defined as grade 3 to 5 of the Louisiana state university health science center scale [10]. Garazzo (2004) recommended associating nerve repair and tendon transfer in one single operative step. (62) cases, over period 15 years, operative management when no spontaneous recovery occurred (3- 4 months), in 53 cases of CPN lesions managed by nerve repair associated with tendone transfer for 2 years follow up, $90 \%$ of cases showed nerve regeneration with dramatic improved in dorsiflexion of the foot [10]. Adolfo (2008):operated on 16 cases with traumatic complete peroneal nerve palsy managed by double tendone, tibialis posterior transferred to tibialis anterior rerouted to new insertion on the third cuneiform and flexor digitorum longus to extensor digitorum longus and extensor hallucis longus and results were excellent in 8 cases, 5 cases were good, two fair and poor in one case [10] Julia A. Seidel (2008) managed 36 cases of CPN lesion with 18 months follow up in grafted group only (28\%) provided functional useful result depend on the graft length, a graft length less than $6 \mathrm{cms}$ leads to function outcome (44\%) with (11) when graft length more than $6 \mathrm{cms}$ with good function outcomes were resulted in cases managed by external neurolysis (73\%), group of interfasicular neurolysis $(71 \%)$. IN 6 patients muscle- tendone transfer were done with strong, useful results of foot dorsiflexion [1]. Heik Reichl et al (2013) common peroneal nerve reconstruction is indicated up to 6 months, they described excision of non-conducting neuroma and graft of deep and superfascial nerves by grafts from sural nerve which fixed by 9.0 proline sutures delayed reconstruction of CPN after 13 months from injury time with the case was followed up for 30 months and presented with strength grade 5 and regular pattern of gait with 90 degree active dorsiflexion [12]. Bryant et al (2014): managed (12) cases with peroneal nerve palsy with simultaneous tendone transfer and nerve exploration for (7) cases, with results superior function in all patients compared to dorsiflexion in $40 \%$ of cases treated with tendone transfer alone, with $57 \%$ of cases treated with both tendone transfer and nerve repair were able to return to running compared to $20 \%$ with tendone transfer alone [13]. Although in CPN palsy recover spontaneously in most cases, when irreversible damage to the nerve can occur so introduction of the concept of decision primary or primary \& secondary reconstruction intraoperative should be considered. As result of high risk of failure rate with cases of C PN palsy with nerve gap defect more than $6 \mathrm{~cm}$ when managed by sural nerve graft alone so we preferred to proceed directly to TP transfer and sural grafting in one step.

In our study, (42) cases of CPN lesion with age range (5- 52), with follow up range (20- 60 months) with different etiology were managed by external neurolysis in ( 9 cases) their useful motor functional outcome were (100\%), internal neurolysis in $(7$ cases) their useful motor functional outcome were $(6 / 7,85.7 \%)$. (15) cases with nerve gap defect lessthan $6 \mathrm{cms}$ and grafted with sural nerve length range $(3-9 \mathrm{cms})$ with useful function results $(12 / 15,80 \%)$ and (11) cases with nerve gap defect more than 6 $\mathrm{cms}$ and managed by sural nerve grafting length range $(9.5-12 \mathrm{cms})$ and TP transfer in one step with useful function outcome (10/11, $90.9 \%)$. The overall results of all procedures to manage (42) patients of CPN lesions were satisfied and excellent in $(37 / 42,88.1 \%)$.

Surgical procedures (6) cases showed complete regression, (7) cases improved with reduction of neuropathic pain, (3) cases recorded as unchanged neuropathic pain and referred to pain control clinic to manage them $(13 / 16,81.2 \%)$. Significant correlation between age, causative mechanism of injury, time of injury and surgical procedure and motor and sensory recovery $(\mathrm{P}=$ 0.002). Significant results of group I, II. III and nerve gap defect $>6 \mathrm{~cm}$ and $<6 \mathrm{~cm}(\mathrm{p}=0.003)$. Significant results of motor and sensory recovery and age $>18$ years $(\mathrm{P}=0.002)$.

\section{CONClusion}

Drop foot after CPN lesion is a challenging problem to treat so preoperative clinical (age of the patient, mechanism of injury, motor and sensory deficit, time interval between palsy and exploration and if there is complete motor loss without regenerative sign) and preoperative and intraoperative neurophysiological evaluation will help to determine site of lesion and provide selected cases for exploration the decision of primary or primary and TP transfer in one step. In our study we provided excellent improvement in motor and sensory function and no patients 
had deteriorated. To increase useful functional motor and sensory recovery out com in CPN palsy we must deal with motor and sensory units as functional units not as anatomical site of lesion, extensive careful intraoperative neurophysiological monitoring achieved increase in distal reinnervation without loss of existing neural function, intraoperative evaluation of scare tissue and muscle fibrosis which contributed to bad results

Recommendation: To develop the optimal common peroneal nerve surgical management strategy, we recommended early nerve exploration and repair when indicated (to overcome the decreasing number of motor end plat and sensory receptors with time as degeneration so before surgery the target is to provide suble mobile ankle and foot joints, good healthy number of motor end plat and sensory receptors) we recommended resection all neuromas incontiniuty nonfunctional of conduction as were found that better long term recovery with grafting especially with extensive soft tissues damage and scare. and intraoperative decision making for primary nerve or primary nerve repair and secondary tendone transfer reconstruction in one step to provide the patient the best chances of sensory recovery and useful motor functional outcomes.

\section{REFERENCES}

[1] Julia A Seidel, Ralph Koenig and Gregor Antoniadis: surgical treatment of traumatic peroneal nerve lesions. Neurosurgery 62, 3: 664 $-673,(2008)$.

[2] Rolf Birch and george Bonney: surgical disorders of the peripheral nerves, shaper (1) (1-17) ( 1998 ).

[3] Tobias loenz and marco campello : biomechanics of skletal muscles : basic biomechanics muscluskeletal system, third edition, chapter 6, 149:171 ( 2001 )

[4] Bjorn Rydevik and Goran lund borg : Biomechanics of peripheral nerves and spinal nerve roots, Basic Biomechanics of the muscloskeletal system, third edition chapter 5 : 127 - 146 ( 2001 ).

[5] James G. and Ross Todd: Biomechanics of the foot and ankle, Basic Biomechanics of the muscloskeletal system, third edition chapter 9 : $230-255$ ( 2001 ) .

[6] Kimura : electrodiagno sis nin diseases of nerves and muscles, oxford university press, $\mathrm{P}$. $128: 131$ (2013)

[7] NIZAR Souayah and josef G poian : motor unit numer estimation as predictior of motor dysfunction animal type diabetes : jully AMG physiol endocrinol, 297 ( 3 ) : $602: 608$ ( 2009 )

[8] Rania S Nagib, Waffaa S Mohamed and Taghreed M Azmy : Role of superfissial peronial sensory potential and high resolution ultrasonography in confermation of common peronial mono neuropathy at fibular neck, the Egyptian jurnal of neurology, psychiatry and neurosurgery 23 March ( 2019 ).

[9] Kim DH, Murovic JA: mangment and outcomes 318 operative common peroneal nerve lesion at the Louisiana state university health science centre.Neurosurgery 54 :14211429 (2004) .

[10] D .Garozzo, S Ferraresi, P .Buffatti : surgical treatment of common peroneal nerve injuries : indication and results . a series of 62 cases . J Neurogsci, volume 48, pp. 105-112 (2004).

[11] Adolfo Vigasio ,Ignazio Marcoccio :new tendone transferefor correction of drop foot in common peroneal nerve palsy . clin orthop relat res, volume 466,number 6 , Jun (2008).

[12] Heike Reich, Florian Ensat : successful delayed recnstruction of common peroneal neuroma in continuity using sural nerve graft. microsurgery, volume 33, 160- 163 (2013).

[13] Bryant Ho, Zubair Khan, George Ochenjele :treatment of peroneal nerne injuries with simultaneous teondone transfer and nerve exploration , journal of orthopaedic surgery and researsh 9,67, (2014).

Citation: Mohamed Abd-El Aziz Mohamed Ali, Mohammed Mansour, Nagy Fouda. Primary Nerve Repair with or without Tibialis Posterior Transfer for Management of Common Peroneal Nerve Palsy. ARC Journal of Orthopedics. 2020; 5(1):6-13. doi:dx.doi.org/ 10.20431/2456-0588.0501002.

Copyright: (C) 2020 Authors. This is an open-access article distributed under the terms of the Creative Commons Attribution License, which permits unrestricted use, distribution, and reproduction in any medium, provided the original author and source are credited. 